RUNNING HEAD: MOVING RHI IN THE MIRROR

\title{
New reflections on agency and body ownership: The moving rubber hand illusion in the mirror
}

Paul M. Jenkinson ${ }^{1 *} \&$ Catherine Preston ${ }^{2}$

${ }^{1}$ Department of Psychology, University of Hertfordshire, Hatfield, Hertfordshire, UK.

${ }^{2}$ Department of Neuroscience, Karolinska Institute, Sweden.

*Correspondence concerning this article should be addressed to: P. M. Jenkinson, Department of Psychology, University of Hertfordshire, College Lane, Hatfield, AL10 9AB. Tel: +44 (0)1707 284618. Email: p.jenkinson@herts.ac.uk.

Word count (excluding abstract, figures/tables \& references) $=6,064$ 


\section{Acknowledgements}

The authors are grateful to Jon Gillard and Noel Taylor for providing technical support on this project, and to Ashley Longhurst for assistance with data collection. We also wish to thank Marco Bertamini and the anonymous second reviewer for their helpful comments on this work. 


\begin{abstract}
No previous study has simultaneously examined body ownership and agency in healthy subjects during mirror self-observation. We used a moving rubber hand illusion to examine how both body ownership and agency are affected by seeing (i) the body moving in a mirror, compared with (ii) directly viewing the moving hand, and (iii) seeing a visually identical hand rotated by $180^{\circ}$. We elicited ownership of the hand using direct visual feedback, finding this effect was further enhanced when looking at the hand in a mirror, whereas rotating the hand $180^{\circ}$ abolished ownership. Agency was similarly elicited using direct visual feedback, and equally so in the mirror, but again reduced for the $180^{\circ}$ hand. We conclude that the reflected body in a mirror is treated as 'special' in the mind, and distinct from other external objects. This enables bodies and actions viewed in a mirror to be directly related to the self.
\end{abstract}

Keywords: mirror; agency; ownership; rubber hand illusion; embodiment; self. 


\section{Introduction}

What happens when we look in a mirror $?^{1}$ In a basic, physical sense, looking in a mirror provides a simple tool for observing one's outer appearance by reflecting light according to geometric rules (Prinz, 2013). Humans are one of only a few animals who can recognise themselves in a mirror, and as such mirror self-recognition is regarded as a litmus test of self-awareness (Amsterdam, 1972; Gallup, 1970; Plotnik, de Waal, \& Reiss, 2006; Reiss, \& Marino, 2001). However, we do not use mirrors to just passively recognise ourselves; we regularly perform complex, mirror-guided actions such as shaving, applying make-up, and brushing our teeth. We are able to perform these intricate actions without consideration, or even awareness, of the many unique properties of mirror images (see Bertamini \& Parks, 2005; Bianchi, Savardi, \& Bertamini, 2008; Lawson \& Bertamini, 2006) and thus the motor transformations required.

In cognitive neuroscience terms, the self that we experience when moving in front of a mirror comprises both a sense of body ownership (i.e. the sense that one's body belongs to oneself) and agency (i.e. the sense that one is the cause or author of one's actions). An abundance of recent research has sought to understand the functional and neuroanatomical mechanisms underlying these fundamental aspects of the self (e.g. Farrer et al., 2003; Jenkinson et al., 2013; Jenkinson, Edelstyn, Preston, \& Ellis, 2014; Kalckert \& Ehrsson, 2012; Newport \& Preston, 2010; Tsakiris, Prabhu, \& Haggard, 2006). However, only a handful of experimental studies have specifically sought to examine the effect of mirror self-observation on body ownership in healthy individuals (see Bertamini, Berselli, Bode, Lawson, \& Wong, 2011; Jenkinson et al.,

\footnotetext{
${ }^{1}$ References to looking in the mirror and mirror reflections through this paper refer to the image produced by a frontally positioned mirror, which is distinct from other research looking at the effect of placing a mirror in the sagittal position
} 
2013; Kontaris \& Downing, 2011), and no study to date has examined the effect of mirror self-observation on agency.

Bertamini et al. (2011) conducted the now classic rubber hand illusion (RHI; Botvinick \& Cohen, 1998), but with the rubber hand observed only in a mirror. They found that a mirror view of the rubber hand elicited strong embodiment, as measured using subjective ratings and perceived drift in location of the real hand towards the rubber hand (i.e. proprioceptive drift). Jenkinson et al. (2013) replicated and extended this effect, finding that the RHI was elicited (as measured using questionnaire ratings), irrespective of whether vision and attention were focused on a directly observed rubber hand, or the specular image of the rubber hand in the mirror. Importantly, in these studies ownership during the mirror RHI was equal to that induced by direct view (but see Kontaris \& Downing, 2011), which contrasts with the finding that ownership is not elicited when the rubber hand is rotated by 180 -degrees and placed in the same location as the specular image (such that the visual properties are identical to that of the mirror condition; see Bertamini et al., 2011; and Kontaris \& Dowling, 2011). These findings show how a strong sense of ownership is experienced when seeing our body in a mirror, and support the unique representation of mirror reflections in the mind.

However, the question of how agency is affected by looking in a mirror has never been specifically and directly examined. A case report of immediate, complete and permanent remission of chronic anosognosia for hemiplegia (i.e. unawareness of paralysis) following video-feedback provides striking evidence of the possible effect that viewing the body from a third-person perspective (similar to that produced from looking in a mirror) can have on (disturbed) agency (Fotopoulou, Rudd, Holmes, \& Kopelman, 2009). However, the mechanism by which this dramatic effect was 
obtained is unclear, with the change in awareness potentially arising as a result of viewing the body 'from the outside', from observing motor performance 'offline' (i.e. when there was no intention to perform a movement), or from a combination of these two factors.

Importantly, both agency and perspective taking have been proposed as critical components of self-consciousness (Vogeley \& Fink, 2003); however, there has been little attempt to investigate the interaction of these two factors in healthy individuals (see David et al., 2006, for an exception). The third-person/observer view of our body (such as that provided by mirror and video feedback) may be important for the sense of agency, given that the ability to discriminate between self and other actions allows us not only to recognise ourselves and guide our own actions, but also to interpret the actions of others (Preston \& Newport, 2010). Alternatively, controlling the body in the mirror may be more similar to using a tool, and so distinct from feelings of agency over the actual body (i.e. external vs. body agency; see Kalckert \& Ehrsson, 2012).

Owing to the absence of any existing experimental evidence, this study specifically set out to examine the effect of mirror self-observation on agency, whilst also attempting to replicate existing findings concerning body ownership. We used an existing method: the moving rubber hand illusion (mRHI; Kalckert \& Ehrsson, 2012, 2014a, 2014b), during which participants observe movements of a model hand while their own hand is hidden from view. The model hand can be moved either actively (under the control of the participant) or passively (under the control of the experimenter), with the movements made by the model and real hand being either synchronous or asynchronous. Using this method, Kalckert and Ehrsson (2012) found that asynchronous movement or rotating the model hand by 180 degrees abolished the 
illusion of ownership, and that ownership was higher during active compared with passive synchronous movements. Agency, on the other hand, was still experienced even for an anatomically impossible, rotated hand. Extending this method to the current experiment, healthy participants performed the mRHI whilst looking at a model hand either directly, rotated 180 degrees, or via a mirror. We expected results of the directly viewed and rotated conditions to replicate those of Kalckert and Ehrsson (2012), as described above. We expected that, in the mirror, ownership and agency would be greater during synchronous as compared with asynchronous movement, and active as compared with passive movement overall. In addition, we predicted that synchronous movements observed in the mirror would produce body ownership equal to that of directly observed movements (see Bertamini et al., 2011; Jenkinson et al., 2013), whereas a $180^{\circ}$ view would abolish ownership. For agency in the mirror, we expected only active and synchronous movement to elicit a sense of agency, and for all three views to produce the same effect (based on the previous finding that a $180^{\circ}$ view does not abolish agency).

We measured the illusion using both subjective (embodiment questionnaire) and objective (proprioceptive drift) measures. In addition to drift in the perceived height of the real hand towards the model hand (Kalckert \& Ehrsson, 2012, 2014a, 2014b; hereafter referred to as "height drift") we also measured drift away from the body / towards the mirror (hereafter referred to as "forward drift"). Objects viewed in a mirror are treated as being located in peripersonal space, despite the image appearing in extrapersonal space (Maravita, Spence, Clarke, Husain, \& Driver, 2000). Therefore, we predicted that despite the projected image being distant from the body (in extrapersonal space), the hand would still be perceived as located in peripersonal space. We therefore expected that the above predictions would be demonstrated via 
changes in height drift and subjective ratings, whereas the forward drift would show no difference across conditions, and was used as a control for demand characteristics and general susceptibility.

\section{Method}

\subsection{Participants}

Thirty-two healthy volunteers (11 male, 21 female; mean age $=23.50, \mathrm{SD}=4.36$; range $=18-32$ years) from the University of Hertfordshire participated in the experiment. All participants were right-hand dominant (Edinburgh Handedness Inventory; Oldfield, 1971) and naïve to the purpose of the experiment. The study was approved by an institutional research ethics committee and all participants provided written informed consent.

\subsection{Design}

The experiment assessed whether Synchrony, Movement type and View influence ownership and agency of the hand. Synchrony between the movements of the real and model hand was manipulated so that movements were either temporally congruent (i.e. no delay between real and model hand movement) or incongruent (i.e. delayed) by approximately $500 \mathrm{~ms}$. Movement type was manipulated so that movements of the two hands were either self-generated by the participant (i.e. active) or generated by the experimenter (i.e. passive). Finally, View was manipulated so that participants were provided with either (i) a direct view of the rubber hand in the canonical position with fingers pointing away from the participant's body, (ii) a view of the hand rotated $180^{\circ}$, with fingers pointing towards the participant's body, or (iii) a view of the (canonically positioned) hand via a mirror (creating visual feedback of the hand with fingers pointing towards the participant). The experiment therefore had a 2 (Synchrony: 
synchronous vs. asynchronous) x 2 (Movement type: active vs. passive) x 3 (View: direct vs. $180^{\circ}$ vs. mirror) design. A fully-factorial combination of these three factors produced the 12 conditions summarised in Table 1. Conditions were completed within-subject in a random order.

\subsection{Materials and set-up}

The experimental materials and set-up replicated an established moving rubber hand illusion method (see Kalckert and Ehrsson, 2012, 2014a, 2014b), with minor modifications to accommodate the mirror conditions (see Design and Procedure). The experiment involved a $16 \mathrm{~cm}(\mathrm{~h}) \times 30 \mathrm{~cm}(\mathrm{w}) \times 45 \mathrm{~cm}(\mathrm{~d})$ box positioned on a table immediately in front of the participant, in alignment with the sagittal body midline (see Figure 1a-c). A model (wooden) artist hand (measuring $30.5 \mathrm{~cm}$ from tip of the middle finger to the base of the wrist) with articulated joints was placed with the palm faced down on top of the box either in a canonical (anatomically congruent) position, or rotated by $180^{\circ}$ (anatomically incongruent). During the mirror conditions direct view of the model hand (placed in the canonical position) was obscured by positioning a piece of cardboard between the model hand and participant's face, and a $40 \mathrm{~cm}(\mathrm{~h}) \times 30 \mathrm{~cm}(\mathrm{w})$ single plane mirror was placed on the box in front of the participant at the point where the fingertips of the model hand ended. The mirror was positioned such that it provided an image identical to that of the $180^{\circ}$ condition, with only the model hand (and not the face of the participant) observable in the mirror. This was achieved by covering the top half of the mirror with a sheet of plane white paper and rotating the angle of the mirror until the participant report being able to see only the model hand. A right model hand was used during the direct and mirror view conditions, whereas a left hand was used during the $180^{\circ}$ conditions, in order to create 
visual feedback matching that of the mirror condition (i.e. a mirror changes an object into its enantiomorph, so a virtual [mirror] image of a right hand appears as if it is a left hand). The participant's own right hand was located directly below the model hand in a relaxed, slightly curled position. White cotton gloves were placed on the model hand and worn on the participant's right hand throughout the experiment in order to control for differences in visual and tactile feedback that might influence the illusion. Participants also wore a dark-coloured cape around their neck, which covered the right side of their body and the distal end of the model hand so that it appear to be an extension of the participant's own right arm. Movement of the model hand was achieved by a thin wooden dowel, one end of which could be attached to the index finger of the model hand via a removable plastic ring (when required; see Procedure). The dowel extended into the box so that the other end of the dowel could be attached to the participant's right index finger with an identical plastic clip (when required; see procedure). An opening on the left side of the box allowed the experimenter (but not the participant) to observe the real hand and dowel mechanism, in order to attach / detach the dowel from the finger and control / monitor movements of the two hands as required by the various experimental conditions (see Design and Procedure). Previous research using this set-up indicates that participants typically do not develop explicit awareness of the mechanism used to produce the observed movements (see Kalckert \& Ehrsson, 2012, p.4). The participant's left hand was located in a resting position with the palm facing down on the table throughout the experiment.

\subsection{Illusion measures}

2.4.1. mRHI Questionnaire 
To assess the subjective experiences of agency and ownership we used the short form of the mRHI questionnaire from Kalckert and Ehrsson (2012). The questionnaire (see supplementary materials) contained two statements to assess ownership (i.e. "related to the experience of perceiving the hand as the 'own' hand"; Kalckert \& Ehrsson, 2014a, p. 120) and two statements measuring agency (i.e. "related to the experience of voluntary control"; Kalckert \& Ehrsson, 2014a, p. 120). A further four statements (2 ownership control and 2 agency control), resembled the illusion-specific statements in wording but did not capture the phenomenological experience of agency or ownership. These served as controls for compliance, suggestibility, and expectancy effects (see Kalckert \& Ehrsson, 2012 \& 2014a for details). During the experiment each statement was presented in a pseudorandom order on each occasion, and rated on a 7-point Likert scale $(-3=$ totally disagree, $0=$ uncertain,$+3=$ totally agree $)$. A visual version of the Likert scale (printed on A4 paper) accompanied the verbal presentation of each statement, and was used to facilitate responses where necessary.

\subsubsection{Proprioceptive drift}

An objective, behavioural measure of the illusion was also obtained by assessing the degree to which the perceived position of the right index finger shifted towards the model hand during each condition (i.e. drift towards the model hand). Proprioceptive drift is a well-established method of assessing ownership of the foreign limb (see Botvinick \& Cohen, 1998; Tsakiris \& Haggard, 2005), which is unrelated to subjective agency ratings (see Kalckert \& Ehrsson, 2012). Following the procedure of Kalckert and Ehrsson (2012, 2014a, 2014b), participants indicated (with their eyes closed) the felt location of their right index finger by using their left index finger to rapidly but accurately point towards a piece of graph paper attached to the left side of 
the experimental apparatus. The experimenter marked on the graph paper the position of the fingertip (rounded to the nearest $\mathrm{cm}$ ), allowing the perceived height, and distance of the hand from the body to be measured. We measured drift in height instead of the more conventional (horizontal plane) proprioceptive drift (see Botvinick \& Cohen, 1998), because the real and model hands were positioned in the same location horizontally, thereby eliminating the possibility of any such drift. This method may also have an advantage over the traditional horizontal measurement as it avoids the capturing effect of the body, which may enhance drift scores in the traditional horizontal plane (Preston, 2013). In addition, we measured the extent to which the perceived position of the right index shifted forward, away from the real body and towards the mirror (i.e. forward drift). This served as a control for general compliance, suggestibility, and expectancy effects relating to proprioceptive drift; that is, since objects viewed in a mirror are processed as being located in (peri)personal space (see Maravita et al., 2000), there should be no forward (or backward) drift.

The perceived location of the right index finger was measured pre- and postinduction in each condition, and drift was calculated by subtracting the pre-induction point from the post-induction point. A positive value for the height drift indicated a shift in the perceived location of the real hand towards the model hand. A positive value for the forward drift indicated a shift towards the mirror / away from the body and location of the real hand.

\subsection{Procedure}

The experiment was conducted in a quiet study room at the University of Hertfordshire and took approximately 1.5 hours to complete. Prior to commencing the first experimental condition participants were familiarised with the proprioceptive 
drift procedure (see above), Likert scale used to measure the illusion (i.e. an explanation of the scale was given and understanding of the scale checked), and tapping movement they would be asked to perform during the experiment. The tapping movement comprised flexion and extension of the right index finger (magnitude of finger lift $\sim 6 \mathrm{~cm}$ ) at a rate of $1 \mathrm{~Hz}$. Participants were instructed to avoid a strictly regular pattern by including a quick double-tap at non-regular (jittered) intervals; this procedure is reported to enhance the strength of the illusion (Kalckert \& Ehrsson, 2012; 2014a), and compliance was checked in a pre-experimental training period, during which the experimenter observed the participant's tapping movement, corrected mistakes (e.g. if the participant adopted a regular tapping pattern), and demonstrated if necessary. A metronome was used to aid timing during training, but was not present during the actual experimental conditions.

During each condition the experimenter sat on the left side of the participant, allowing access to the open side of the experimental box. At the start of the condition the model hand (positioned either canonically or $180^{\circ}$ rotated), and mirror (where applicable) were put into position on top of the box, and the real hand placed inside the box (see section 2.2 and table 1). The participant then viewed the model hand for 30s (to control for embodiment as a result of visual capture; see Pavani, Spence, \& Driver, 2000) before completing the pre-induction (pointing) measure of proprioceptive drift. Induction of the illusion was then implemented as described below. In all cases participants viewed the model hand without view of their own right hand or the experimenter's hand.

In the active conditions the participant was instructed to self-generate the practiced tapping movement for $60 \mathrm{~s}$. In passive movement conditions the participant was instructed to relax their hand and the experimenter moved the real and model 
hand for 60 s. For synchronous conditions the real and model hand were connected via the wooden dowel and finger clips so that movement of the two hands were temporally congruent. During asynchronous conditions the finger clip worn on the real hand was detached from the wooden dowel; however, the clip remained on the participant's finger to maintain consistent somatosensory feedback across all conditions. The experimenter then used the dowel inside the box to move the model hand (and real hand in passive conditions) in a way that mimicked movements of the actual hand but with a temporal delay of $\sim 500 \mathrm{~ms}$.

Immediately after the 60 s movement period participants completed the second (post-induction) pointing measure of proprioceptive drift, followed by the $\mathrm{mRHI}$ questionnaire. A rest period of at least $45 \mathrm{~s}$ occurred between each condition, during which the participant was instructed to remove his or her hand from the box and encouraged to flex the fingers and shake the wrist to abolish the previous illusion induction. Following completion of all conditions the participant was thanked, debriefed, and dismissed.

\subsection{Data handling and analysis}

Subjective measures of agency and ownership were calculated by averaging the two agency statements and separately the two ownership statements to produce composite measures of agency and ownership. The control agency and control ownership statements were likewise averaged to produce composite measures of agency control and ownership control. These composite scores were used in subsequent analyses. Our objective measure of ownership (proprioceptive drift) was calculated as outlined above (section 2.4.2). For clarity, in the results below we first 
report our findings regarding the ownership questionnaire and proprioceptive drift, and then our agency findings.

For our statistical analysis we examined each condition's questionnaire and drift data for normality (via visual inspection of histograms, and the KolmogorovSmirnov and Shapiro-Wilk tests), which was violated in the majority of cases ( $p$ $<.05)$; therefore, non-parametric statistics were applied. In two preliminary checks (presented in supplementary materials for brevity) we examined the overall effect of Statement (illusion vs. control) (where applicable) and Synchrony (synchronous vs. asynchronous) on ownership, proprioceptive drift, and agency. We subsequently examined the main effects of Movement Type and View, and Movement Type $\mathrm{x}$ View interaction for each measure. All analyses were implemented using SPSS version 21, using Exact tests where possible. Reported $p$-values are 2-tailed and Bonferroni-corrected as indicated.

\section{Results}

Table 2 summarises the illusion and control statement scores for ownership and agency in each condition.

\subsection{Ownership Ratings}

\subsubsection{Main Effect of Movement type}

The overall effect of movement type was examined irrespective of visual perspective by conducting a Wilcoxon signed ranks test on the mean of all synchronous passive trials compared to all synchronous active trials. Participants reported significantly 
higher ownership for active compared to passive movement $(z=-4.12, p<.001$; see Figure 2a).

\subsubsection{Main Effect of View}

The overall effect of view was examined by calculating the mean ownership score for each view (irrespective of movement type) and comparing these using a Friedman's ANOVA. This revealed a significant difference between ownership ratings: $\chi^{2}(2)=$ 40.62, $p<.001$ (see Figure 2b). Post-hoc comparisons were carried out using Wilcoxon signed ranks tests (Bonferroni-corrected critical $\alpha=.017$ ). Mirror view was found to produce higher ownership scores than both direct $(z=-3.34, p=.001)$ and $180^{\circ}(z=-4.70, p<.001)$ views. Direct view produced significantly higher ownership scores compared to $180^{\circ}$ view $(z=-4.75, p<.001)$.

\subsubsection{Movement type $x$ View interaction}

To examine whether the effect of view is modulated by movement type, we calculated the difference between active and passive scores for each view and conducted a Friedman's ANOVA on these differentials (see Hicks, 1973; Mead, 1988). Results of this analysis indicated no significant interaction between the two factors: $\chi^{2}(2)=5.08$, $p=.078$.

\subsection{Proprioceptive drift}

Table 3 summarises the two measures of proprioceptive drift for each condition.

\subsubsection{Effect of Movement type}


A Wilcoxon signed ranks test comparing the mean of all synchronous passive trials to all synchronous active trials, revealed significantly greater height drift for active movement $(z=-4.09, p<.001$; see Figure $2 \mathrm{c})$. There was no significant difference in forward drift between active and passive movement $(z=-1.23, p=.112)$.

\subsubsection{Effect of View}

A Friedman's ANOVA comparing height drift across views (irrespective of movement type) revealed a significant effect, $\chi^{2}(2)=12.62, p=.001$ (see Figure $2 d$ ). Subsequent pairwise comparisons (using Wilcoxon signed rank tests and a Bonferroni-corrected critical $\alpha=.017$ ), showed less drift in height as a result of the $180^{\circ}$ view compared with both direct $(z=-2.84, p=.005)$ and mirror $(z=-3.18, p$

$=.001)$ views. Direct and mirror views produced equivalent height drift $(z=-1.92, p$ $=.055)$. As expected, there was no effect of view on forward drift, $\chi^{2}(2)=1.71, p$ $=.430$.

\subsubsection{Movement type $x$ View interaction}

A Friedman's ANOVA on the active-passive differential scores of each view (as described in section 3.1.3 above) indicated no interaction between movement type and view on height drift, $\chi^{2}(2)=4.28, p=.116$, or forward drift, $\chi^{2}(2)=2.00, p=.381$.

\subsection{Agency Ratings}

\subsubsection{Effect of Movement type}

A Wilcoxon signed ranks test, comparing the mean of all synchronous passive trials with all synchronous active trials, indicated significantly higher agency ratings during active movement $(z=-4.86, p<.001$; see Figure $3 a)$. 


\subsubsection{Effect of View}

A Friedman's ANOVA comparing agency ratings across views (irrespective of movement type), revealed a significant overall effect, $\chi^{2}(2)=22.66, p<.001$ (see Figure 3b). Subsequent pairwise Wilcoxon signed ranks tests (Bonferroni-corrected $\alpha$ $=.017)$, revealed that $180^{\circ}$ view produced lower agency ratings compared with both direct $(z=-3.53, p=.005)$ and mirror $(z=-3.48, p=.001)$ views. There was no significant difference between direct and mirror views $(z=-.68, p=.496)$.

\subsubsection{Movement type $x$ View interaction}

A Friedman's ANOVA on the active-passive differential scores of each view (as described in section 3.1.2. above) indicated a significant interaction between movement type and view: $\chi^{2}(2)=14.70, p<.001$ (see Figure 3c). To further break down this interaction we compared agency ratings between the direct, mirror, and $180^{\circ}$ view during active movement, and separately during passive movement (using Wilcoxon signed ranks tests and a Bonferroni-corrected critical $\alpha=.008$ ). Results indicated that the interaction arose as a result of differences in agency between views during active movement only, which were not present for passive moment. Specifically, for the active movements mirror and direct view produced significantly greater agency ratings compared with the $180^{\circ}$ view $\left(180^{\circ}\right.$ vs. mirror: $z=-3.37, p$ $=.001$; and $180^{\circ}$ vs. direct: $z=-4.50, p<.001$ ), while there was no significant difference between mirror and direct view ratings $(z=-1.75, p=.08)$. There was no difference in agency ratings across views for passive movements $(\max z=-2.18, p$ $=.03)$. 


\section{Discussion}

In this study we examined how both body ownership and agency are experienced in a mirror. With regards to body ownership, our results support our predictions and previous findings that ownership can be elicited whilst viewing the model hand via a mirror, but not when viewing the hand in a visually identical posture ( $180^{\circ}$ rotation) without a mirror (Bertamini et al., 2011; Ehrsson, Spence, \& Passingham, 2004; Kontaris \& Downing, 2011; Tsakiris \& Haggard, 2005), or when movements of the real and model hand are asynchronous (Kalckert \& Ehrsson, 2012). Moreover, our unique investigation of agency in a mirror revealed that viewing (active synchronous) movements in a mirror elicits equally strong agency as when the model hand is seen directly. By contrast, viewing the hand rotated by $180^{\circ}$ reduces (but does not abolish) agency (see Kalckert \& Ehrsson, 2012 for another example of this latter finding). These novel results suggest that when hand movements are observed in a mirror, they are processed in a similar way to movements of the actual body, as opposed to an external object. These findings suggest that mirrors are treated as 'special' in the mind, thereby enabling bodies and actions viewed in a mirror to be directly related to the self.

Interestingly, subjective reports of ownership were higher when looking at the hand in the mirror compared to directly. This finding is contrary to those of previous studies that found statistically equivalent (Bertamini et al., 2011; Jenkinson et al., 2013) or reduced (Kontaris \& Downing, 2011) ownership in a mirror; however, the observed enhancement of body ownership in the mirror is consistent with neuropsychological studies which find that mirror self-observation can reinstate body ownership in patients with somatoparaphrenia (Fotopoulou et al., 2011). How can we explain this enhancement effect? We consider two, possibly related factors that may 
be important: firstly, we used a moving version of the rubber hand illusion in the current study, whereas previous research has examined body ownership in the mirror using only the classic (static) rubber hand illusion. Thus, body ownership might be enhanced during active movements because efferent motor signals may contribute to the feeling of ownership (discussed in more detail below). Furthermore, even during passive movements, the moving rubber hand illusion involves more possible channels of sensory information (e.g. cutaneous afferents, muscle spindle receptors and joint receptors), which are not engaged by tactile stimulation in the classic rubber hand illusion (Kalckert \& Ehrsson, 2014a). Despite such differences, the classic and moving rubber hand illusions have been found to elicit similarly strong feelings of ownership (Kalckert \& Ehrsson, 2014a); however, in our study the mirror may have led certain signals to be considered more important than others, resulting in the observed enhancement of ownership.

This differential weighting of certain sensorimotor signals might occur as a result of increased sensorimotor 'noise', or imprecision, created by discrepancies between the location of the felt and seen body when looking in the mirror (see Fotopoulou, 2014, for further discussion of the role played by sensorimotor noise in the construction of the bodily self). Existing research indicates that the specular image of the body seen in a mirror is treated as being located in peripersonal space (Maravita et al., 2000). Accordingly, if the hand in the mirror is treated as being in peripersonal space (as part of the body) as opposed to the position that it appears visually in space, then the brain must compute a transformation relating the events seen in the mirror to the position of the felt body. This transformation may introduce additional sensory noise, such that small discrepancies in hand appearance and location are less noticeable, or given less weight, when judging ownership. Feelings of ownership are 
consequently increased in a mirror relative to direct view. Indeed, introducing noise to the visual feedback of reaches has been found to reduce discrepancy detection of self and other movements (Preston \& Newport, 2014) and the introduction of a mirror may increase noise in a similar way.

On the other hand, feelings of agency were not enhanced in the mirror view condition. This may be linked to the fact that during synchronous movement agency judgments were more binary in nature compared to ownership; synchronous active movement produced strong feelings of agency $($ median $=+3)$ in both direct and mirror view, whereas synchronous passive movement showed the opposite extreme in responses. The proposed effect of the extra transformation from mirror view may, therefore, only be evident in conditions where there is more ambiguity or uncertainty in sensorimotor signals and consequently greater response variability. For example, asynchronous movement creates much greater conflict or noise between sensory and motor signals, and although significantly reducing feelings of agency, does not completely abolish agency (as does passive movement). Therefore, examining asynchronous conditions can eliminate the statistical issues involved with ceiling and floor effects that are apparent for the synchronous conditions. In fact, post hoc analysis of the asynchronous active conditions indicates that mirror view elicits significantly stronger agency compared to direct view $(z=-2.64, p=.008)$, which further supports the facilitatory effect of viewing the hand in a mirror. However, the exact mechanisms underlying this effect, and why it is absent in previous mirror rubber hand illusion studies that do not have ceiling and floor effects, is unclear. Future research should therefore aim to replicate and directly examine the mechanisms behind enhanced illusion scores in a mirror. In particular, the role played by sensorimotor noise, and the contradictory findings that mirror self-observation can 
both impair (Paysant et al., 2004) and improve body ownership (Fotopoulou et al., 2011; Jenkinson et al., 2013) following brain injury deserve further attention.

Our results also largely replicated those described by Kalckert and Ehrsson (2012), who demonstrated evidence for experimental double dissociation between agency and ownership. As well as agency and ownership being dissociable, however, they are also found to interact (Kalckert \& Ehrsson, 2012, 2014a; Tsakiris et al., 2006). In the current results, even though agency was not abolished completely whilst viewing the hand from $180^{\circ}$ (median score of 2 in the active condition) it was significantly lower compared to direct view. This finding supports that previously described by Kalckert and Ehrsson (2012) who interpreted the effect as representing a difference between body agency and non-body agency, such that feelings of agency are stronger when it is your own body moving as opposed to an external object under one's control. Unfortunately, we did not include a non-hand control condition in our design, so we cannot rule out the possibility that a sense of agency might also be experienced when a non-hand object moves in synchrony with one's motor intentions. Future studies should therefore also examine agency over a non-body object seen in the mirror in order to clarify the relationship between body and non-body (i.e. external) agency.

Importantly, our results did show enhanced agency during mirror view (compared to $180^{\circ}$ ). If, as suggested by the ownership results, mirror view produces feelings that the seen hand is part of the body, enhanced agency in mirror view compared to $180^{\circ}$ may also reflect body agency. Therefore, mirror modulation of agency may also be driven at least in part by ownership. Indeed post hoc correlations (Spearman's rho) between agency and ownership (synchronous - asynchronous scores) in the active conditions reveal the two to be strongly related for both direct $\left(r_{2}\right.$ 
$=.58, p=.001)$ and mirror $\left(r_{2}=.58, p<.001\right)$ view, but not $180^{\circ}\left(r_{2}=.35, p=.047\right)$ (critical alpha $=.017$ following Bonferroni correction). Therefore, these novel findings provide further support for the interactive nature of agency and ownership, and may suggest that the beneficial effects of mirror observation on agency related disorders, such as anosognsosia for hemiplegia, might work in part via their interaction with body ownership mechanisms. This helps to explain why mirrors appear to be more effective in treating ownership disorders such as somatoparaphrenia, while having a seemingly less dramatic and enduring effect on agency-related conditions (Fotopoulou, personal communication); however, further neuropsychological studies with robust assessment of ownership and agency during mirror self-observation are needed to further substantiate this idea.

Finally, results from the current study also provide evidence for an enhancing effect of efference signals on the experience of ownership, for both direct and mirror view. Significantly higher scores were found for active compared to passive movements in the ownership questionnaire responses as well as proprioceptive (height) drift. This is contrary the findings of Kalckert and Ehrsson (2012) who, despite finding differences in subjective reports of ownership, found statistically equivalent proprioceptive drift with both active and passive movements. Interestingly, for the current results there was also no significant effect of synchrony on passive movements, suggesting that passive movements failed to cause remapping of the perceived hand location. Although proprioceptive drift is a widely used objective measure of the illusion, it does not always correspond with subjective feelings of ownership (Holle, McLatchie, Maurer, \& Ward, 2011; Rohde, Di Luca, \& Ernst, 2011) possibly accounting for inconsistent results in the literature. Similarly the effect of active vs. passive movement on the illusion is also found to be inconsistent 
(Dummer, Picot-Annand, Neal, \& Moore, 2009; Kalckert \& Ehrsson, 2012, 2014a; Walsh, Moseley, Taylor, \& Gandevia, 2011). One of the key differences between the current paradigm and that described by Kalckert and Ehrsson (2012) is the trial duration, lasting for 90 s in the original study and only 60 s in the current study. It may be that although passive movement can elicit equivalently strong illusions as active movements, it may take longer to do so. Although $60 \mathrm{~s}$ is a more than sufficient duration to elicit strong feelings of ownership, it may be that remapping of perceived hand location takes longer to establish and longer still when the movements are passive. Future studies should investigate perceived hand location and illusion strength at different time points during illusion induction and whether this is modulated by mirror view.

In conclusion, our findings contribute to the rich body of existing literature, which aims to understand how humans (Amsterdam, 1972) and other animals (Gallup, 1970; Plotnik et al., 2006; and Reiss \& Marino, 2001) recognise and relate to themselves in the mirror (see also Rochat \& Zahavi, 2011, for a critical reappraisal of the mirror self-recognition literature). The current results demonstrate that both direct and mirror visual feedback of a model hand elicit strong feelings of ownership and agency (during active movement). In fact, seeing the hand in a mirror leads to ownership ratings that exceed those arising from directly viewing the hand, suggesting an enhancement of body ownership above typical levels. These results suggest that when a body part is viewed in a mirror, it is treated in a similar way to viewing the body directly. Moreover, the reflection in the mirror, despite providing an objectified view of the body 'from the outside' (rotated by $180^{\circ}$ ), is processed as part of the self and distinct from a visually identical external object. Therefore, the current study supports the notion that bodies viewed in mirrors are treated in a special way, 
perceived as positioned within peripersonal space (self), and that this extends to both action (agency) as well as perception (ownership). 


\section{REFERENCES}

Amsterdam, B. (1972). Mirror self-image reactions before age two. Developmental Psychobiology, 5, 297-305. doi:10.1002/dev.420050403

Bertamini, M., Berselli, N., Bode, C., Lawson, R., \& Wong, L. T. (2011). The rubber hand illusion in a mirror. Consciousness and Cognition, 20(4), 1108-19. doi:10.1016/j.concog.2011.04.006

Bertamini, M., \& Parks, T. E. (2005). On what people know about images on mirrors. Cognition, 98(1), 85-104. doi:10.1016/j.cognition.2004.11.002

Bianchi, I., Savardi, U., \& Bertamini, M. (2008). Estimation and representation of head size (people overestimate the size of their head - evidence starting from the 15th century). British Journal of Psychology, 99(4), 513-31. doi:10.1348/000712608X304469

Botvinick, M., \& Cohen, J. (1998). Rubber hands "feel" touch that eyes see. Nature, 397(6669), 756. doi:10.1038/35784

David, N., Bewernick, B. H., Cohen, M. X., Newen, A., Lux, S., Fink, G. R., ... Vogeley, K. (2006). Neural representations of self versus other: visual-spatial perspective taking and agency in a virtual ball-tossing game. Journal of Cognitive Neuroscience, 18(6), 898-910. doi:10.1162/jocn.2006.18.6.898

Dummer, T., Picot-Annand, A., Neal, T., \& Moore, C. (2009). Movement and the rubber hand illusion. Perception, 38(2), 271-280. doi:10.1068/p5921 
Ehrsson, H., Spence, C., \& Passingham, R. (2004). That's my hand! Activity in premotor cortex reflects feeling of ownership of a limb. Science, 305(5685), 875-7. doi:10.1126/science.1097011

Farrer, C., Franck, N., Georgieff, N., Frith, C. . D., Decety, J., \& Jeannerod, M. (2003). Modulating the experience of agency: A positron emission tomography study. NeuroImage, 18(2), 324-333. doi:10.1016/S1053-8119(02)00041-1

Fotopoulou, A. (2014). The virtual bodily self: Mentalisation of the body as revealed in anosognosia for hemiplegia. Consciousness and Cognition. doi:10.1016/j.concog.2014.09.018

Fotopoulou, A., Jenkinson, P. M., Tsakiris, M., Haggard, P., Rudd, A., \& Kopelman, M. D. (2011). Mirror-view reverses somatoparaphrenia: Dissociation between first- and third-person perspectives on the body. Neuropsychologia, 49, 39463955. doi:10.1016/j.neuropsychologia.2011.10.011

Fotopoulou, A., Rudd, A., Holmes, P., \& Kopelman, M. (2009). Self-observation reinstates motor awareness in anosognosia for hemiplegia. Neuropsychologia, $47(5), 1256-60$.

Gallup, G. G. (1970). Chimpanzees: Self-recognition. Science, 167, 86-87. doi: 10.1126/science.167.3914.86

Hicks, C. R. (1973). Fundamental Concepts in the Design of Experiments. New York, NY: Holt, Rinehart and Winston. 
Holle, H., McLatchie, N., Maurer, S., \& Ward, J. (2011). Proprioceptive drift without illusions of ownership for rotated hands in the "rubber hand illusion" paradigm. Cognitive Neuroscience, 2(3-4), 171-8. doi:10.1080/17588928.2011.603828

Jenkinson, P. M., Edelstyn, N. M. J., Preston, C., \& Ellis, S. J. (2014). Anarchic hand with abnormal agency following right inferior parietal lobe damage: a case report. Neurocase, 1-8. doi:10.1080/13554794.2014.925936

Jenkinson, P. M., Haggard, P., Ferreira, N. C., \& Fotopoulou, A. (2013). Body ownership and attention in the mirror: insights from somatoparaphrenia and the rubber hand illusion. Neuropsychologia, 51(8), 1453-62.

doi:10.1016/j.neuropsychologia.2013.03.029

Kalckert, A., \& Ehrsson, H. H. (2012). Moving a Rubber Hand that Feels Like Your Own: A Dissociation of Ownership and Agency. Frontiers in Human Neuroscience, 6(March), 40. doi:10.3389/fnhum.2012.00040

Kalckert, A., \& Ehrsson, H. H. (2014a). The moving rubber hand illusion revisited: comparing movements and visuotactile stimulation to induce illusory ownership. Consciousness and Cognition, 26, 117-32. doi:10.1016/j.concog.2014.02.003

Kalckert, A., \& Ehrsson, H. H. (2014b). The spatial distance rule in the moving and classical rubber hand illusions. Consciousness and Cognition, 30, 118-132. doi:10.1016/j.concog.2014.08.022

Kontaris, I., \& Downing, P. E. (2011). Reflections on the hand: The use of a mirror highlights the contributions of interpreted and retinotopic representations in the rubber-hand illusion. Perception, 40(11), 1320-1334. doi:10.1068/p6825 
Lawson, R., \& Bertamini, M. (2006). Errors in judging information about reflections in mirrors. Perception, 35, 1265-1288. doi:10.1068/p5498

Maravita, A., Spence, C., Clarke, K., Husain, M., \& Driver, J. (2000). Vision and touch through the looking glass in a case of crossmodal extinction. NeuroReport, $11,3521-3526$.

Mead, R. (1988). The design of experiments. Cambridge: Cambridge University Press.

Newport, R., \& Preston, C. (2010). Pulling the finger off disrupts agency, embodiment and peripersonal space. Perception, 39, 1296-1298. doi:10.1068/p6742

Oldfield, R. C. (1971). The assessment and analysis of handedness: The Edinburgh inventory. Neuropsychologia, 9, 97-113.

Pavani, F., Spence, C., \& Driver, J. (2000). Visual capture of touch: out-of-the-body experiences with rubber gloves. Psychological Science, 11, 353-359. doi:10.1111/1467-9280.00270

Paysant, J., Belis, J. M., Le Chapelain, L., Andr, , J. M., Beis, J. M., \& André, J. M. (2004). Mirror asomatognosia in right lesions stroke victims. Neuropsychologia, 42(7), 920-925. doi:10.1016/j.neuropsychologia.2003.12.001

Plotnik, J. M., de Waal, F. B. M., \& Reiss, D. (2006). Self-recognition in an Asian elephant. Proceedings of the National Academy of Science, 103, 17053-17057. doi: 10.1073/pnas.0608062103 
Preston, C. (2013). The role of distance from the body and distance from the real hand in ownership and disownership during the rubber hand illusion. Acta Psychologica, 142(2), 177-83. doi:10.1016/j.actpsy.2012.12.005

Preston, C., \& Newport, R. (2010). Self-denial and the role of intentions in the attribution of agency. Consciousness and Cognition, 19, 986-998. doi:10.1016/j.concog.2010.04.005

Preston, C., \& Newport, R. (2014). Noisy visual feedback training impairs detection of self-generated movement error: implications for anosognosia for hemiplegia. Frontiers in Human Neuroscience, 8(June), 456. doi:10.3389/fnhum.2014.00456

Prinz, W. (2013). Self in the mirror. Consciousness and Cognition, 49, 1-9. doi:10.1016/j.concog.2013.01.007

Reiss, D., \& Marino, L. (2001). Mirror self-recognition in the bottlenose dolphin: A case of cognitive convergence. Proceedings of the National Acadamy of Science, 98, 5937-5942. doi: 10.1073/pnas.101086398

Rochat, P., \& Zahavi, D. (2011). The uncanny mirror: A reframing of mirror selfexperience. Consciousness and Cognition, 20(2), 204-213. doi:10.1016/j.concog.2010.06.007

Rohde, M., Di Luca, M., \& Ernst, M. O. (2011). The Rubber Hand Illusion: feeling of ownership and proprioceptive drift do not go hand in hand. PloS One, 6(6), e21659. doi:10.1371/journal.pone.0021659 
Tsakiris, M., \& Haggard, P. (2005). The rubber hand illusion revisited: visuotactile integration and self-attribution. Journal of Experimental Psychology. Human Perception and Performance, 31(1), 80-91. doi:10.1037/0096-1523.31.1.80

Tsakiris, M., Prabhu, G., \& Haggard, P. (2006). Having a body versus moving your body: How agency structures body-ownership. Consciousness and Cognition, 15(2), 423-32. doi:10.1016/j.concog.2005.09.004

Vogeley, K., \& Fink, G. R. (2003). Neural correlates of the first-person-perspective. Trends in Cognitive Sciences, 7(1), 38-42.

Walsh, L. D., Moseley, G. L., Taylor, J. L., \& Gandevia, S. C. (2011). Proprioceptive signals contribute to the sense of body ownership. The Journal of Physiology, 589(12), 3009-21. doi:10.1113/jphysiol.2011.204941. 


\section{FIGURES}

(a)

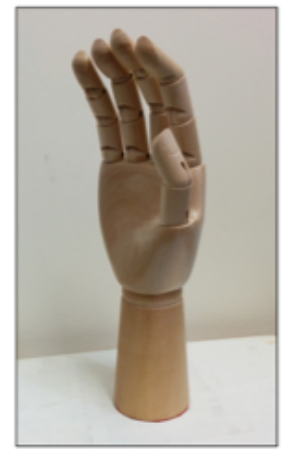

(b)

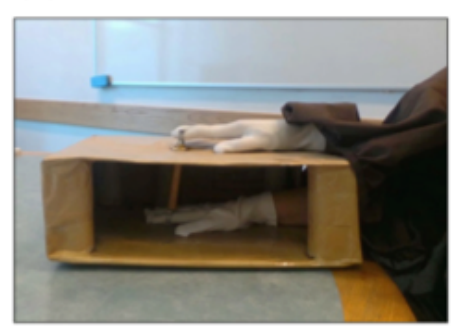

(c)

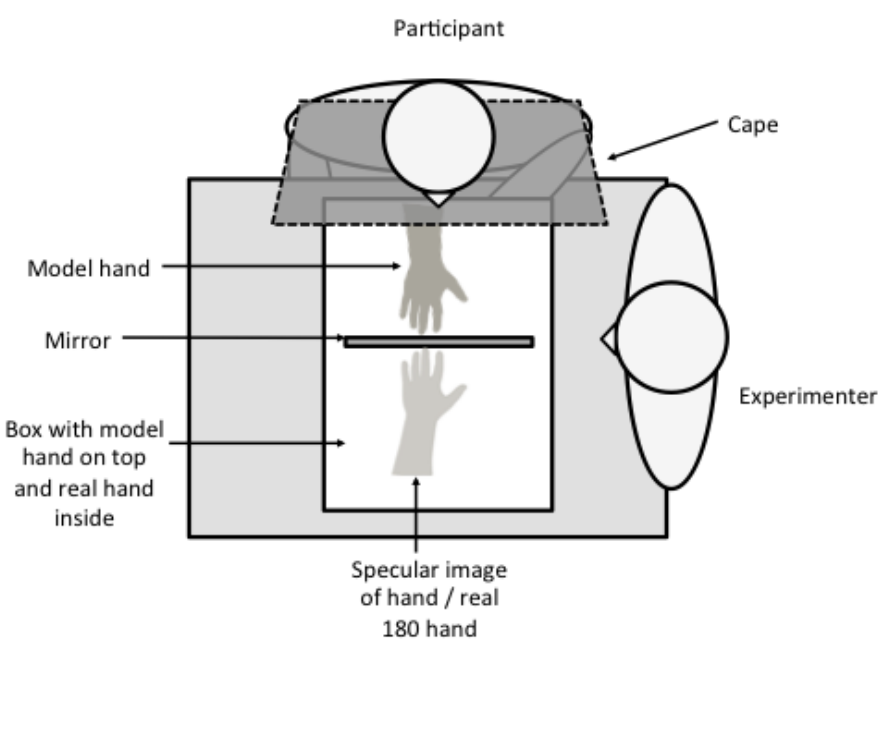

Figure 1. The experimental setup, showing the model hand (panel A), side view of the box with the right model hand on top and real hand inside (panel B), and overall arrangement of apparatus (panel C). During direct view conditions the model right hand was placed on top of the box. During mirror view conditions a mirror was placed immediately in front of the model hand and positioned such that only the reflection of the model hand could be seen. During $180^{\circ}$ view conditions a model left hand was placed in the same location as the specular image created by the model right hand. 

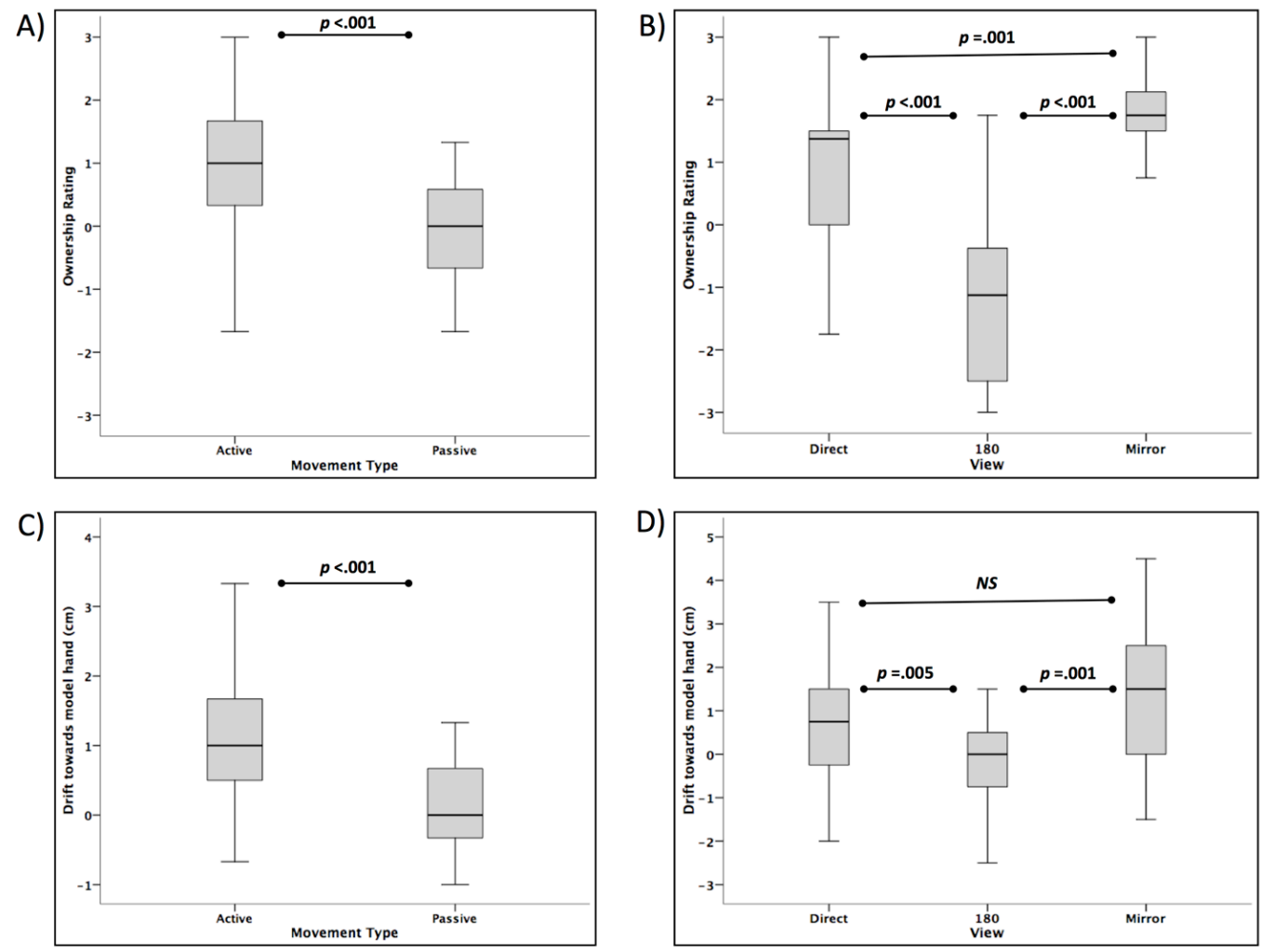

Figure 2. Main effect of Movement Type (left panels) and View (right panels) for ownership ratings (top panels) and proprioceptive drift (bottom panels). Thick black lines indicate the median. The box indicates the interquartile range, and the whiskers indicate the range. 
A)

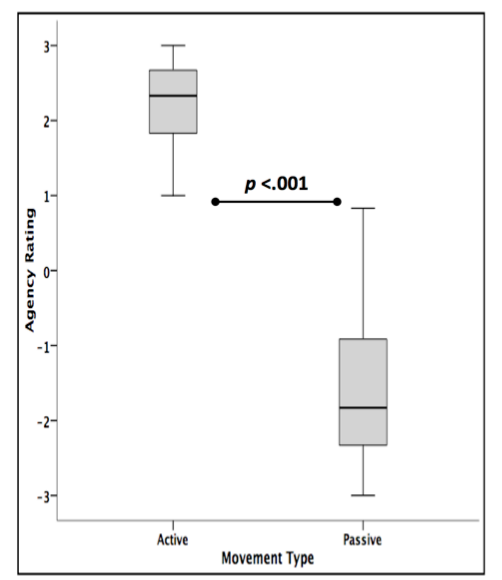

B)

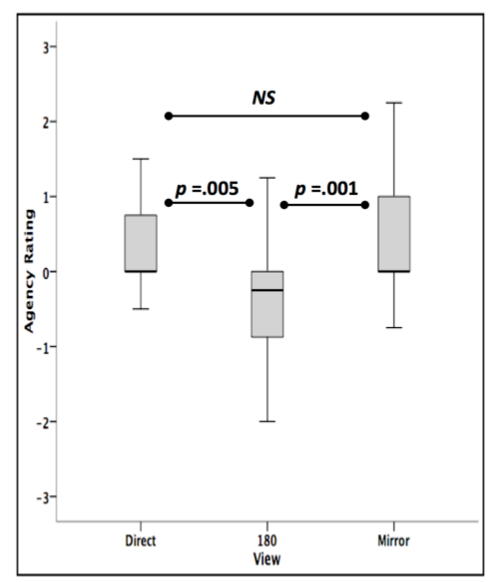

C)

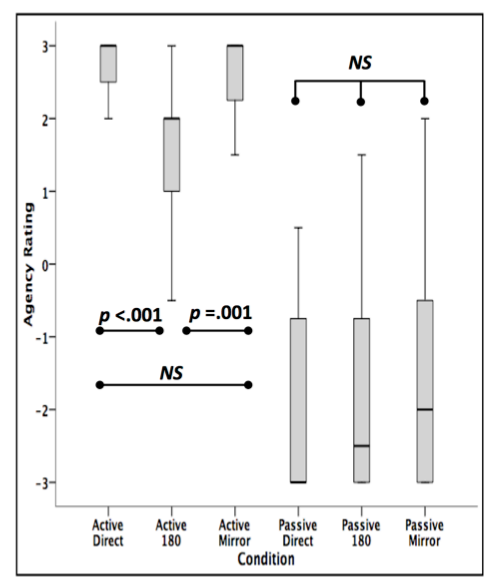

Figure 3. Main effect of Movement Type (panel A), View (panel B), and Movement Type $\mathrm{x}$ View interaction (panel C) for agency ratings. Thick black lines indicate the median. The box indicates the interquartile range, and the whiskers indicate the range. 